PROCEEDINGS OF THE

AMERICAN MATHEMATICAL SOCIETY

Volume 130, Number 10, Pages 3085-3089

S 0002-9939(02)06403-1

Article electronically published on March 13, 2002

\title{
A RAMSEY THEOREM FOR MEASURABLE SETS
}

\author{
M. LACZKOVICH
}

(Communicated by Carl G. Jockusch, Jr.)

\begin{abstract}
We prove that if $X$ is a perfect Polish space and $[X]^{2}=P_{0} \cup \ldots \cup$ $P_{k-1}$ is a partition with universally measurable pieces, then there is Cantor set $C \subset X$ with $[C]^{2} \subset P_{i}$ for some $i$.
\end{abstract}

By a theorem of F. Galvin, if $X$ is a perfect Polish space and $[X]^{2}=P_{0} \cup \ldots \cup P_{k-1}$ is a partition with pieces having the Baire property, then there is Cantor set $C \subset X$ with $[C]^{2} \subset P_{i}$ for some $i$. (See [4], [5], and [6] 19.7, p. 130].) Our aim is to prove the analogous statement for universally measurable sets.

Theorem 1. Let $X$ be a nonempty perfect Polish space and $[X]^{2}=P_{0} \cup \ldots \cup P_{k-1}$ is a partition, where each $P_{i}$ is universally measurable in the sense that $P_{i}^{*}=$ $\left\{(x, y) \in X^{2}:\{x, y\} \in P_{i}\right\}$ is universally measurable. Then there is a Cantor set $C \subset X$ with $[C]^{2} \subset P_{i}$ for some $i$.

Note that the statement is not true for infinitely many pieces or for $[X]^{3}$ instead of $[X]^{2}$ (see [6, 19.9 and 19.10]). We shall prove Theorem 1 by presenting a sufficient condition for the existence of "squares" (sets of the form $P \times P$ ) contained in a given subset of $\mathbf{R}^{2}$. By a theorem of M. L. Brodskii [1], every subset of $\mathbf{R}^{2}$ of positive measure contains the product of two perfect sets (see also [3, p. 114]). Of course, a set of positive measure need not contain squares. As the trivial example $\{(x, y):|y-x|>\varepsilon\}$ suggests, in order to find squares in a set $H \subset \mathbf{R}^{2}$ we need an extra condition saying that $H$ is large in the vicinity of the diagonal $\Delta=\{(x, x)$ : $x \in \mathbf{R}\}$. We remark that no reasonable condition implies the existence of a subset $P \times P$ where $P$ is a perfect set of positive measure (take $H=\{(x, y): x-y$ is irrational $\})$. Moreover, as Z. Buczolich showed 2 there is a set $H$ of full measure such that whenever $P$ and $Q$ are closed sets such that $P \times Q \subset H$ and $P$ is of positive measure, then the Hausdorff dimension of $Q$ is zero.

We shall use the following notation. If $H \subset \mathbf{R}^{2}$, then we shall denote $H^{*}=$ $\{(x, y):(y, x) \in H\}$. The set $H$ will be called symmetric if $H^{*}=H$. The sections of $H$ are denoted by $H_{x}=\{y:(x, y) \in H\}$ and $H^{y}=\{x:(x, y) \in H\}$. The Lebesgue outer measure in $\mathbf{R}^{k}$ will be denoted by $\lambda_{k}$. The symmetric upper density of the set $A \subset \mathbf{R}$ at the point $x \in \mathbf{R}$ is defined by

$$
\bar{d}(A, x)=\limsup _{h \rightarrow 0+} \frac{\lambda_{1}(A \cap(x-h, x+h))}{2 h} .
$$

Received by the editors February 2, 2000 and, in revised form, May 17, 2001.

2000 Mathematics Subject Classification. Primary 03E02, 28A05.

(C)2002 American Mathematical Society 
The symmetric lower density $\underline{d}(A, x)$ is defined by taking the lim inf of the same quotient.

Theorem 2. Let $H \subset \mathbf{R}^{2}$ be a symmetric Lebesgue measurable set satisfying

$$
\lambda_{1}\left(\left\{x \in \mathbf{R}: \bar{d}\left(H_{x}, x\right)>0\right\}\right)>0 .
$$

Then there is a nonempty perfect set $P \subset \mathbf{R}$ such that $P \times P \subset H \cup \Delta$.

Proof of Theorem 2. First we note that if $H \subset \mathbf{R}^{2}$ is measurable, then, by Fubini's theorem, the function $f_{h}$ defined by $f_{h}(x)=\lambda_{1}\left(H_{x} \cap[x-h, x+h]\right) \quad(x \in \mathbf{R})$ is also measurable for every fixed $h$. Therefore the functions $\bar{f}(x)=\bar{d}\left(H_{x}, x\right)$ and $\underline{f}(x)=\underline{d}\left(H_{x}, x\right)$ are measurable as well, since $\bar{f}=\limsup _{n \rightarrow \infty} f_{1 / n} \cdot n / 2$ and $\bar{f}=\liminf _{n \rightarrow \infty} f_{1 / n} \cdot n / 2$.

Now we show that if $H \subset \mathbf{R}^{2}$ is closed, symmetric and satisfies (1), then there is a nonempty perfect set $P$ such that $P \times P \subset H$.

If $n$ is a positive integer and $n=2^{k} m$, where $m$ is odd, then we shall denote $r(n)=k$. Then $0 \leq r(n)<n$ for every $n>0$. Also, for every nonnegative integer $k$ there are infinitely many $n$ 's with $r(n)=k$.

It is well-known that for every measurable $H \subset \mathbf{R}^{2}$ there is a set $A \subset H$ such that $\lambda_{2}(H \backslash A)=0$, and for every $(x, y) \in A, x$ is a density point of $A^{y}$ and $y$ is a density point of $A_{x}$ (see [7, pp. 130-131]). If $H$ is symmetric, then $A$ can also be chosen symmetric, since otherwise we take $A \cap A^{*}$ instead of $A$. Suppose that $H$ is a symmetric closed set satisfying (1), and let $A$ be a symmetric subset of $H$ with the property described above. Since $\lambda_{2}(H \backslash A)=0$, it follows from Fubini's theorem that $\lambda_{1}\left((H \backslash A)_{x}\right)=0$ for a.e. $x$, and hence $\bar{d}\left(H_{x}, x\right)=\bar{d}\left(A_{x}, x\right)$ for a.e. $x$. Then the set $D=\left\{x: \bar{d}\left(A_{x}, x\right)>0\right\}$ is measurable and has positive measure. Let $D^{0}$ denote the set of those elements of $D$ which are density points of $D$. We shall define a sequence $x_{n} \in D^{0}$ as follows. Let $x_{0} \in D^{0}$ be arbitrary. Let $n>0$ and suppose that $x_{i} \in D^{0}$ has been selected for every $i<n$ such that $\left(x_{i}, x_{j}\right) \in A$ for every $i, j<n, i \neq j$. Then $x_{r(n)} \in A_{x_{i}}$ for every $i<n, i \neq r(n)$ and, consequently, $x_{r(n)}$ is a density point of the set $E=\bigcap\left\{A_{x_{i}}: i<n, i \neq r(n)\right\}$. Since $x_{r(n)} \in D^{0}, x_{r(n)}$ is also a density point of $D^{0}$. Finally, as $x_{r(n)} \in D$, it follows from the definition of $D$ that the outer density of $A_{x_{r(n)}}$ at $x_{r(n)}$ is positive. This implies that the outer density of the set

$$
M=E \cap A_{x_{r(n)}} \cap D^{0}=\bigcap\left\{A_{x_{i}}: i<n\right\} \cap D^{0}
$$

at $x_{r(n)}$ is positive. In particular, $x_{r(n)}$ is a point of accumulation of $M$. Let $x_{n} \in M$ be such that $0<\left|x_{n}-x_{r(n)}\right|<1 / n$. Then $x_{n} \in D^{0}$ and $\left(x_{i}, x_{n}\right) \in A$ for every $i<n$.

In this way we have defined $x_{n}$ for every $n$. The set $S=\left\{x_{n}: n=0,1, \ldots\right\}$ is dense in itself, since for every $k$ there are infinitely many $n$ 's with $r(n)=k$ and for these $n$ 's we have $0<\left|x_{n}-x_{k}\right|<1 / n$. Let $P$ be the closure of $S$, then $P$ is perfect. Since $(S \times S) \backslash \Delta \subset A \subset H$ and $H$ is closed, it follows that $P \times P \subset H$.

Finally, we prove that if $H$ is measurable, symmetric and satisfies (1), then $H \cup \Delta$ contains a symmetric closed subset which also satisfies (1); this will finish the proof of the theorem.

Put $H_{n}=\left\{(x, y) \in H: x-\frac{1}{n} \leq y \leq x+\frac{1}{n}\right\}$, and let $F_{n}$ be a closed subset of $H_{n}$ such that $\lambda_{2}\left(H_{n} \backslash F_{n}\right)<n^{-4} \quad(n=1,2, \ldots)$. We define $F_{0}=\bigcup_{n=1}^{\infty} F_{n}$ and $F=F_{0} \cup F_{0}^{*} \cup \Delta$. Then $F$ is closed, symmetric, and is contained in $H \cup \Delta$. We 
shall prove that $\bar{d}\left(F_{x}, x\right)=\bar{d}\left(H_{x}, x\right)$ for a.e. $x$. Since $\lambda_{1}\left(\left\{x: \bar{d}\left(H_{x}, x\right)>0\right\}\right)>0$ by assumption, this will prove that $F$ also satisfies (1). Let

$$
G_{n}=\left\{x \in \mathbf{R}: \lambda_{1}\left((H \backslash F)_{x} \cap\left[x-\frac{1}{n}, x+\frac{1}{n}\right]\right)>n^{-2}\right\} .
$$

By $\lambda_{2}\left(H_{n} \backslash F_{n}\right)<n^{-4}$ we have $\lambda_{1}\left(G_{n}\right)<n^{-2}$. Thus $\sum_{n=1}^{\infty} \lambda_{1}\left(G_{n}\right)<\infty$ and hence the set $G=\bigcap_{k=1}^{\infty} \bigcup_{n=k}^{\infty} G_{n}$ is of measure zero. If $x \notin G$, then, for $n$ large enough,

$$
\lambda_{1}\left((H \backslash F)_{x} \cap\left[x-\frac{1}{n}, x+\frac{1}{n}\right]\right) \leq n^{-2} .
$$

Therefore $\bar{d}\left((H \backslash F)_{x}, x\right)=0$ and $\bar{d}\left(F_{x}, x\right)=\bar{d}\left(H_{x}, x\right)$, which completes the proof.

Proof of Theorem 1. We may assume that $X$ is a Cantor set. Applying a suitable homeomorphism, we may also suppose that $X$ is a closed subset of $\mathbf{R}$ having positive measure. The sets $P_{i}^{*}$ are symmetric, and, being universally measurable, they are also measurable with respect to the Lebesgue measure. If $x$ is a density point of $X$, then

$$
1=\bar{d}(X, x) \leq \sum_{i=0}^{k-1} \bar{d}\left(\left(P_{i}^{*}\right)_{x}, x\right),
$$

and thus $\max _{0 \leq i<k} \bar{d}\left(\left(P_{i}^{*}\right)_{x}, x\right)>0$. Therefore $\lambda_{1}\left(\left\{x \in \mathbf{R}: \bar{d}\left(\left(P_{i}^{*}\right)_{x}, x\right)>0\right\}\right)>0$ holds for at least one of $i=0 \ldots k-1$. For such an $i$ the set $P_{i}^{*}$ satisfies the condition of Theorem 2. Therefore $P \times P \subset P_{i}^{*} \cup \Delta$ for a suitable nonempty perfect $P$, and thus $[P]^{2} \subset P_{i}$.

It is obvious that the condition of symmetry cannot be omitted from Theorem 2 (consider the set $\{(x, y): y>x\}$ ). For nonsymmetric sets we can prove the following.

Theorem 3. If $H \subset \mathbf{R}^{2}$ is measurable and

$$
\lambda_{1}\left(\left\{x \in \mathbf{R}: \underline{d}\left(H_{x}, x\right)>1 / 2\right\}\right)>0,
$$

then there is a nonempty perfect set $P$ such that $P \times P \subset H \cup \Delta$.

Proof. First we show that if (2) holds, then the set $E=H \cap H^{*}$ satisfies the following condition: $E$ is symmetric and there is an interval $I$ such that

$$
\limsup _{h \rightarrow 0+} \lambda_{2}(\{(x, y): x \in I, y-h \leq x \leq y+h\} \cap E) / h>0 .
$$

Indeed, (2) implies that there is an $\varepsilon>0$ and there is an interval $I$ such that the set $B=\left\{x \in I: \underline{d}\left(H_{x}, x\right)>\frac{1}{2}+\varepsilon\right\}$ is of positive measure. As we saw in the proof of Theorem 2, $B$ is measurable. We put $T(h)=\{(x, y): x \in B, \quad x-h \leq y \leq x+h\}$ for every $h>0$. Then $T(h)^{*}=\{(x, y): y \in B, y-h \leq x \leq y+h\}$. We prove that

$$
\lim _{h \rightarrow 0+} \lambda_{2}\left(T(h)^{*} \backslash T(h)\right) / h=0 .
$$

If $A \subset \mathbf{R}^{2}$ is measurable, then $\lambda_{2}(A)=\int_{-\infty}^{\infty} \lambda_{1}(\{x:(x, x+t) \in A\}) d t$ by Fubini's theorem. Since

$$
\left\{x:(x, x+t) \in T(h)^{*} \backslash T(h)\right\}=(B-t) \backslash B
$$


for every $|t| \leq h$, we have

$$
\lambda_{2}\left(T(h)^{*} \backslash T(h)\right)=\int_{-h}^{h} \lambda_{1}((B-t) \backslash B) d t .
$$

Now $\lambda_{1}((B-t) \backslash B) \rightarrow 0$ as $t \rightarrow 0$, and (4) follows. It is easy to see that

$$
\liminf _{h \rightarrow 0+} \lambda_{2}(T(h) \cap H) /(2 h) \geq\left(\frac{1}{2}+\varepsilon\right) \lambda_{1}(B),
$$

and thus

$$
\liminf _{h \rightarrow 0+} \lambda_{2}\left(T(h)^{*} \cap H^{*}\right) /(2 h) \geq\left(\frac{1}{2}+\varepsilon\right) \lambda_{1}(B) .
$$

Since

$$
\begin{aligned}
\lambda_{2}(H & \left.\cap H^{*} \cap T(h) \cap T(h)^{*}\right) \\
& =\lambda_{2}(H \cap T(h))+\lambda_{2}\left(H^{*} \cap T(h)^{*}\right)-\lambda_{2}\left((H \cap T(h)) \cup\left(H^{*} \cap T(h)^{*}\right)\right) \\
& \geq \lambda_{2}(H \cap T(h))+\lambda_{2}\left(H^{*} \cap T(h)^{*}\right)-\lambda_{2}(T(h))-\lambda_{2}\left(T(h)^{*} \backslash T(h)\right),
\end{aligned}
$$

it follows from (4), (5), and (6) that

$$
\liminf _{h \rightarrow 0+} \lambda_{2}\left(H \cap H^{*} \cap T(h) \cap T(h)^{*}\right) /(2 h) \geq(1+2 \varepsilon) \lambda_{1}(B)-\lambda_{1}(B)-0>0 .
$$

Therefore, $E=H \cap H^{*}$ satisfies (3), even if the limsup is replaced by liminf .

Now we prove that $\lambda_{1}\left(\left\{x: \bar{d}\left(E_{x}, x\right)>0\right\}\right)>0$. By Theorem 2 , this implies that $E \cup \Delta$ (and thus $H \cup \Delta$ as well) contains a set of the form $P \times P$, where $P$ is nonempty perfect. By (3), there is a $\delta>0$ such that

$$
\lambda_{2}\left(\left\{(x, y): x \in I, y-h_{n} \leq x \leq y+h_{n}\right\} \cap E\right)>\delta h_{n} \quad(n=1,2, \ldots)
$$

for a suitable sequence of positive numbers $h_{n}$ converging to zero. Let

$$
\eta=\delta /(2 \max (|I|, 1))
$$

and

$$
C_{n}=\left\{x \in I: \lambda_{1}\left(E_{x} \cap\left[x-h_{n}, x+h_{n}\right]\right)>\eta h_{n}\right\} .
$$

Then $C_{n}$ is measurable for every $n$. Also, we have $\lambda_{1}\left(C_{n}\right)>\delta / 4$ since otherwise $\lambda_{1}\left(C_{n}\right) \leq \delta / 4$ would imply, by Fubini's theorem,

$$
\lambda_{2}\left(\left\{(x, y): x \in I, y-h_{n} \leq x \leq y+h_{n}\right\} \cap E\right) \leq \frac{\delta}{4} \cdot 2 h_{n}+\frac{\delta h_{n}}{2|I|} \cdot|I|=\delta h_{n},
$$

which is impossible. Let $C=\bigcap_{k=1}^{\infty} \bigcup_{n=k}^{\infty} C_{n}$. Then $\lambda_{1}(C) \geq \delta / 4>0$, and for every $x \in C$ there are infinitely many $n$ 's with $x \in C_{n}$. Therefore $\bar{d}\left(E_{x}, x\right) \geq \eta / 2>0$ for every $x \in C$, completing the proof.

\section{REFERENCES}

[1] M. L. Brodskii, On some properties of sets of positive measure (Russian), Uspekhi Mat. Nauk. 4, No. 3, 31 (1949), 136-139. MR 11:18a

[2] Z. Buczolich, Product sets in the plane, sets of the form $A+B$ on the real line and Hausdorff measures, Acta Math. Hungar. 65 (1994), no. 2, 107-113. MR 95g:28016

[3] H. G. Eggleston, Two measure properties of Cartesian product sets, Quart. J. Math. Oxford (2) 5 (1954), 108-115. MR 16:344e

[4] F. Galvin, Partition theorems for the real line, Notices Amer. Math. Soc. 15 (1968), 660.

[5] F. Galvin, Errata to "Partition theorems for the real line", Notices Amer. Math. Soc. 16 (1969), 1095. 
[6] A. S. Kechris: Classical Descriptive Set Theory. Graduate Texts in Mathematics No. 156. Springer, 1995. MR 96e:03057

[7] S. Saks: Theory of the Integral. Dover, 1965. MR 29:4850

Department of Analysis, EÖtvös Loránd University, Budapest, PÁZmány PÉter sétàny 1/C, 1117 Hungary - and - Department of Mathematics, University College London, Gower Street, London, WC1E 6BT, England

E-mail address: laczko@renyi.hu 